\title{
РАСЧЕТ ПАРАМЕТРОВ СТРУЙНОГО АППАРАТА ДЛЯ ОПТИМАЛЬНОГО РЕЖИМА СОВМЕСТНОЙ РАБОТЫ С УСТАНОВКОЙ ЭЛЕКТРОЦЕНТРОБЕЖНОГО НАСОСА
}

\author{
Сарачева Диана Азатовна',
}

sarachevadiana85@mail.ru

\author{
Вахитова Роза Ильгизовна', \\ roza-w@mail.ru
}

\author{
Уразаков Камил Рахматуллович², \\ UrazakK@mail.ru \\ 1 Альметьевский государственный нефтяной институт, \\ Россия, 423450, г. Альметьевск, ул. Ленина, 2. \\ 2 Уфимский государственный нефтяной технический университет, \\ Россия, 450062, г. Уфа, ул. Космонавтов, 1.
}

\begin{abstract}
Актуальность исследования обусловлена необходимостью решения проблемы удаления газа, скапливающегося в затрубном пространстве нефтедобывающих скважин, на приеме погружного электроцентробежного насоса. Избыточное количество свободного газа в пространстве между обсадной колонной и насосно-компрессорными трубами способствует образованию гидратных пробок, снижению динамического уровня в скважине, в результате чего может наступить срыв подачи и возможна полная остановка добычи нефти. Для откачки свободного газа предлагается использовать струйный аппарат при совместной эксплуатации с электроцентробежным насосом, при этом важно правильно рассчитать место расположения эжектора в скважине и диаметр сопла.

Цель: разработать и предложить методику расчета параметров струйного аппарата для оптимального режима совместной работы с электроцентробежным насосом.

объект: струйный аппарат для удаления затрубного газа в скважинах, оборудованных установками электроцентробежных насосов и имеющих высокий газовый фактор.

Методы. Поставленная проблема решалась на базе статистического анализа промысловых данных с применением информационных современных методов обработки результатов. Использован метод математического моделирования при расчете основных параметров струйного аппарата для обеспечения оптимального режима при их совместной работе с установками электроцентробежных насосов.

Результаты. Предложена математическая модель комплектования электроцентробежного насоса совместно со струйным аппаратом, предназначенным для откачки затрубного газа, в результате этого получены аналитические зависимости, определяющие оптимальные параметры и расчетные величины струйного аппарата, обеспечивающие устойчивую работу системы «электроцентробежный насос - струйный аппарат». Разработана методика расчета места расположения эжектора в скважине, эксплуатируемой установкой электроцентробежного насоса.
\end{abstract}

\section{Ключевые слова:}

Струйный аппарат, газожидкостная смесь, затрубное пространство, электроцентробежный насос, свободный газ, эжектор, пластовая жидкость, насосно-компрессорные трубы.

В процессе эксплуатации нефтедобывающих скважин с применением электроцентробежного погружного насоса (ЭЦН) в последнее время для увеличения продуктивности добычи пластовой жидкости используются в общей комплектации эжекторы [1-4]. Эжекторы, входящие в состав установки ЭЦН, предназначены для отвода из затрубного пространства скопившегося газа (предотвращение образования гидратов, улучшение характеристик динамического уровня и рабочих давлений). При этом важно правильно выбрать и установить струйный аппарат по отношению к погружному насосу и устью скважины, это позволит увеличить добычу нефти и снизить возможность срывов подачи $[5,6]$.

На рис. 1 представлена общая схема расположения погружной установки ЭЦН и струйного аппарата, предназначенного для отбора свободного газа из межтрубного пространства добывающих скважин. Пластовая жидкость вместе с растворенным или свободным газом поднимается к приему погружного насоса от забоя скважины, и в затрубное пространство сепарируется ограниченный объем газа. Газожидкостная смесь, газовая фаза в которой частично представлена растворенным в нефти газом, частично - не отсепарированным на приеме свободным газом, протекает через ЭЦН и затем по насосно-компрессорным трубам (НКТ) поднимается к приему струйного аппарата. Пластовая жидкость, попадая в сопло струйного аппарата, ускоряется, в приемной камере создается область пониженного давления, и газ из затрубного пространства нефтяной скважины устремляется в струйный аппарат. Внутри аппарата газожидкостная смесь (ГЖ⿱С) поступает в камеру смешения, а далее направляется на устье скважины.

В зависимости от технологических характеристик нефтедобывающей скважины (коэффициент 
продуктивности, обводненность, давление пласта, газовый фактор) проводится расчет оптимальных параметров струйного аппарата (глубина спуска и диаметр сопла).

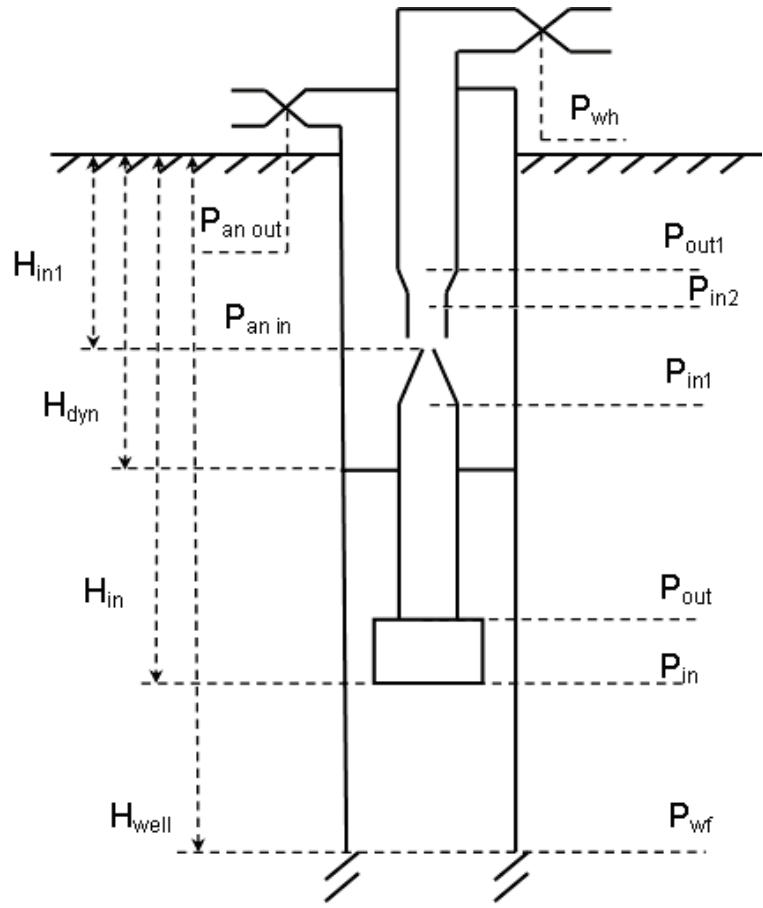

Pис.1. Схема расположения струйного аппарата в погружной установке: $P_{\text {wh }}-$ давление на уровне устья; $P_{\text {anout }}-$ устье вое давление скважины в затрубном пространстве; $P_{\text {out1 }}$ - давление газожидкостной смеси в диффузоре; $P_{\text {in2 }}$ давление на входе в приемной камере; $P_{\text {anin }}-$ давление ско пившегося газа в затрубном пространстве; $P_{\text {in } 1}-$ давле ние пластовой продукции у входа в струйный аппарат; $P_{\text {out2 }}$ - давление газожидкостной смеси на выходе из каме ры смешения; $P_{\text {out }}-$ давление на выходе из насоса; $P_{\text {in }}-\partial a$ вление на приеме погружного насоса: $P_{\text {wf }}-$ пластовое да вление; $H_{\text {in1 }}$ - глубина установки струйного аппарата; $H_{\text {dyn }}-$ динамический уровень; $H_{\text {in }}$ - глубина спуска погружного насоса; $H_{\text {шеll }}$ гллубина скважины

Fig. 1. Layout of the jet apparatus in the submersible installation: $P_{\text {wh }}$ is the wellhead pressure; $P_{\text {anout }}$ is the pressure at the wellhead level in the annulus; $P_{\text {out } 1}$ is the pressure of gas-liq uid mixture in the diffuser; $P_{\mathrm{in} 2}$ is the inlet pressure in the re ceiving chamber; $P_{\text {anin }}$ is the accumulated gas pressure in the annulus; $P_{\text {in1 }}$ is the pressure of reservoir production at the en trance to the jet apparatus; $P_{\text {out }}$ is the pressure of the gas-liq uid mixture at the outlet of the mixing chamber; $P_{\text {out }}$ is the pressure at the outlet of the pump; $P_{\text {in }_{2}}$ is the submersible pump inlet pressure; $P_{\mathrm{wf}}$ is the reservoir pressure; $H_{\text {in } 1}$ is the depth of installation of the jet apparatus; $H_{\mathrm{dyp}}$ is the dynamic level; $H_{\text {in }}$ is the depth of descent submersible pump; $H_{\text {well }}$ is the well depth

Для эффективной работы данной установки необходимо, чтобы при постоянном поступлении скопившегося газа в струйный аппарат обеспечивалось условие превышения давления в затрубном пространстве на глубине установки струйного аппарата $P_{a n \text { in }}$ над давлением в приемной камере [7]. В противном случае из затрубного пространства будет невозможен процесс эжекции газа, а эжектор, находящийся в составе погружной установки, создавая дополнительную разницу давлений на участке НКТ в диапазоне своего расположения, должен будет осуществлять роль штуцера. Поэтому важно рассчитать распределение давления в стволе скважины и в струйном аппарате.

На рис. 2 [8] представлено типичное поведение графических зависимостей производительности погружной установки и притока пластовой жидкости. Рабочие характеристики нефтяной скважины определяет точка пересечения кривых, при этом местонахождение кривой производительности погружной установки зависит от рабочих основных параметров струйного аппарата и ЭЦН, от коэффициента сепарации скопившегося газа на приеме электроцентробежного насоса, а также от месторасположения струйного аппарата относительно приема ЭЦН.

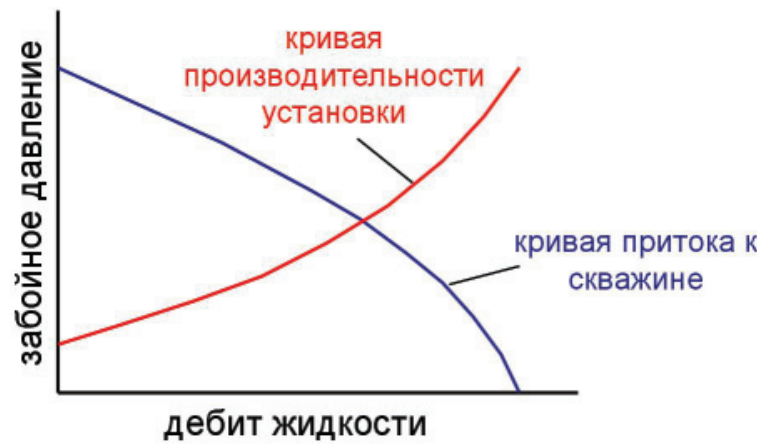

Puс. 2. Графические зависимости погружной установки

Fig. 2. Graphic dependencies of submersible installation

Для нахождения рабочей точки требуется методика для определения на протяженных участках нефтедобывающей скважины разницы давлений в струйном аппарате и погружном насосе.

При определении перепада давления в цилиндрических трубах используем модель Ансари [9], позволяющую спрогнозировать градиент давления в нефтяной скважине для различающихся режимов течения. Для нахождения градиента давления в затрубном пространстве применим метод Кабира и Хасана [10].

Для моделирования перепада давления в погружной установке построим общую модель установки, которая не ориентирована на конкретное оборудование. Предположим, что электроцентробежный насос обеспечит определенный напор:

$$
H=\frac{P_{\text {out }}-P_{\text {in }}}{\rho_{\text {wat }} g D_{\text {gas }} D_{\text {vis }}} \leq H_{c r},
$$

где $P_{\text {in }}$ и $P_{\text {out }}$ - давления на приеме и выкиде погружного насоса, Па; $D_{v i s}$ и $D_{g a s}-$ коэффициенты деградации напорной характеристики из-за вязкости и наличия газа соответственно; $H_{c r}$ и $\mathrm{H}$ - номинальный максимально допустимый и фактический напор насоса, м; $\rho_{\text {wat }}$ - плотность воды, принимаемая равной $1000 \mathrm{\kappa г} / \mathrm{m}^{3}$.

Рассмотрим схему движения газожидкостного потока в струйном аппарате (рис. 3). Газожидкостная смесь на входе в струйный аппарат с давлени- 
ем $P_{i n 1}$ проходит через сопло и ускоряется. В результате на входе приемной камеры эжектора давление $P_{i n 2}$ понижается. В струйном аппарате при наличии положительной разницы давлений $\left(P_{\text {anin }}-P_{\text {in } 2}\right)$ будет происходить инжекционный процесс скопившегося газа. В процессе калориметрического смешивания скорость и температура пластовой жидкости и инжектированного газа выравниваются. Предположим, что давление газожидкостной смеси после прохождения камеры смешивания соответствует величине $P_{\text {out } 2}$, давление на выходе струйного аппарата равно $P_{\text {out } 1}$.

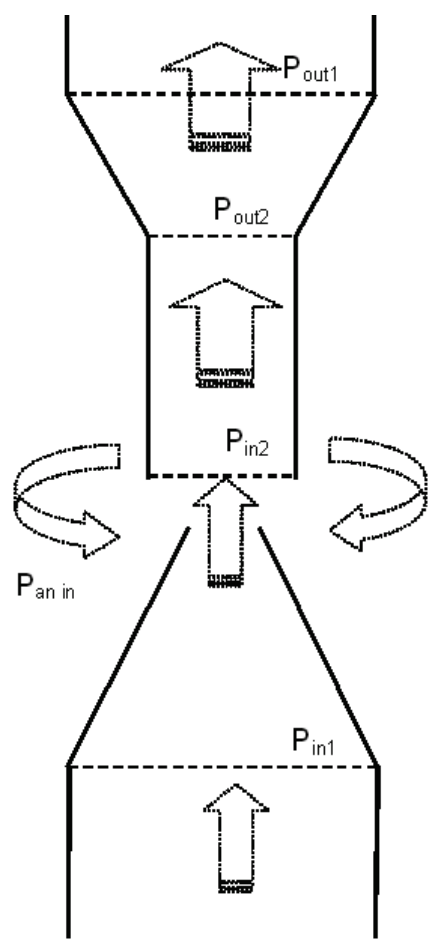

Pис. 3. Схема перепадов давлений в струйном аппарате: $P_{\text {оut1 }}$ давление в диффузоре смеси; $P_{\text {in2 }}-$ давление у входа в при емную камеру; $P_{\text {anin }}$ - давление скопившегося газа в за трубном пространстве; $P_{\mathrm{in1}}-$ давление на входе в струй ном аппарате пластовой жидкости; $P_{\text {оut2 }}$ - давление ГЖС в камере смешения на выходе

Fig. 3. Scheme of pressure drops in the jet apparatus: $P_{\text {out } 1}$ is the pressure in the diffuser mixture; $P_{\text {in } 2}$ is the pressure at the en trance to the receiving chamber; $P_{\text {anin }}$ is the accumulated gas pressure in the annulus; $P_{\text {in } 1}$ is the inlet pressure in the jet ap paratus of the reservoir fluid; $P_{\text {out } 2}$ is the pressure of GLM in the mixing chamber at the outlet

Определим параметры струйного аппарата в сечении $P_{\text {out } 1}$ при заданных показателях пластовой жидкости и инжектируемого газа в сечении $P_{i n 1}$.

Для вычисления давления у входа в приемную камеру эжектора $P_{i n 2}$ предполагаем, что течение в сопловом аппарате пластовой жидкости будет осуществляться вдоль линий тока. Несжимаемая пластовая продукция при этом будет иметь плотность $\rho=2 \rho_{i n 1} \rho_{i n 2} /\left(\rho_{i n 1}+\rho_{i n 2}\right)$. Для решения уравнения сохранения количества движения используем уравнение Бернулли [4]:

$$
\frac{\rho_{i n 1}+\rho_{i n 2}}{2 \rho_{i n 1} \rho_{i n 2}}\left(P_{i n 1}-P_{i n 2}\right)=\left(1+\xi_{i n 2}\right) \frac{u_{i n 2}^{2}}{2}-\frac{u_{i n 1}^{2}}{2},
$$

где $\xi_{\text {in2 }}$ - коэффициент сопротивления сопла; $u_{i n 2}$ и $u_{i n 1}$ - скорости пластовой жидкости в сечении сопла и на входе в струйный аппарат, $\mathrm{m} / \mathrm{c} ; \rho_{i n 1}$ и $\rho_{i n 2}-$ плотности пластовой жидкости в сечениях $P_{i n 1}$ и $P_{i n 2}$.

Закон сохранения массы в процессе протекания пластовой жидкости через сопловый аппарат будет иметь следующий вид:

$$
\rho_{\text {gin } 1} Q_{\text {gin } 1}+\rho_{\text {lin } 1} Q_{\text {lin } 1}=\rho_{\text {gin } 2} Q_{\text {gin } 2}+\rho_{\text {in } 2} Q_{\text {in } 2},
$$

где $\rho_{g}$ и $\rho_{l}-$ плотности газа и жидкости, кг $/ \mathrm{M}^{3} ; Q_{g}$ и $Q_{l}-$ дебиты свободного газа и пластовой жидкости в различных сечениях, $\mathrm{M}^{3} /$ сут.

При этом плотность жидкости рассчитаем по формуле:

$$
\rho_{l}=w c \rho_{w}+(1-w c) \rho_{o},
$$

где $w c$ - объем воды; $\rho_{0}$ и $\rho_{w}$ - соответственно значения плотностей нефти и воды, кг $/ \mathrm{M}^{3}$.

Учитываем, что плотность воды остается постоянной, а плотность пластовой жидкости зависит от температуры и давления. Из уравнения состояния газов Менделеева-Клайперона определяем плотность свободного газа:

$$
\rho_{\text {с.г. }}=\rho_{g} R T,
$$

где $T$ - температура, ${ }^{\circ} \mathrm{K} ; R$ - универсальная газовая постоянная, Дж/(кг·К).

При одинаковых значениях температуры и давления пластовой жидкости и свободного газа плотность смеси определяется с учетом плотности фаз:

$$
\rho=\frac{\rho_{g} Q_{g}+\rho_{l} Q_{l}}{Q_{g}+Q_{l}} .
$$

Для определения изменения температурных параметров в сечении $\left(P_{i n 1}-P_{i n 2}\right)$ воспользуемся уравнением сохранения полной энергии газожидкостной смеси:

$$
\begin{aligned}
& \rho_{\text {gin } 1} Q_{g i n 1}\left(c_{v g} T_{i n 1}+\frac{P_{i n 1}}{\rho_{\text {gin } 1}}+\frac{u_{i n 1}^{2}}{2}\right)+ \\
& +\rho_{\text {lin } 1} Q_{\text {lin } 1}\left(c_{v l} T_{i n 1}+\frac{P_{i n 1}}{\rho_{\text {lin } 1}}+\frac{u_{i n 1}^{2}}{2}\right)= \\
& =\rho_{\text {gin } 2} Q_{g i n 2}\left(c_{v g} T_{i n 2}+\frac{P_{i n 2}}{\rho_{\text {gin } 2}}+\frac{u_{i n 2}^{2}}{2}\right)+ \\
& +\rho_{\text {lin } 2} Q_{\text {lin } 2}\left(c_{v l} T_{i n 2}+\frac{P_{i n 2}}{\rho_{\text {lin } 2}}+\frac{u_{i n 2}^{2}}{2}\right) .
\end{aligned}
$$

Дебит добывающей скважины и скорость движения пластовой жидкости зависимы друг от друга:

$$
u=\frac{4 Q}{\pi d^{2}},
$$

где $d$ - диаметр НКТ, м.

С помощью системы уравнений (1)-(7) можно определить в сечении $P_{i n 2}$ параметры струйного ап- 
парата. Аналогичные системы уравнений можно записать и для сечений $\left(P_{\text {out } 2}-P_{\text {out } 1}\right)$ и $\left(P_{\text {an in }}-P_{\text {in } 1}\right)$. Обобщенная форма уравнения Бернулли для потока реальной жидкости требует учета коэффициента, характеризующего неравномерность распределения скоростей по сечению потока. В общем случае значение коэффициента зависит от формы эпюры (профиля) скорости и может значительно варьироваться. В связи с вышесказанным в качестве уравнения сохранения количества движения для данной области записывается закон сохранения импульса в виде:

$$
\begin{gathered}
\left(P_{\text {in } 2}-P_{\text {out } 2}\right) S_{\text {out } 2}+ \\
+\rho_{\text {gin } 2}\left(u_{\text {in } 2} Q_{\text {gin } 2}+u_{\text {in } 2}^{\prime} Q_{\text {gin } 2}^{\prime}\right)+\rho_{\text {in } 2} u_{\text {in } 2} Q_{\text {in } 2}= \\
=\rho_{\text {gout } 2} u_{\text {out } 2} Q_{\text {gout } 2}+\rho_{\text {out } 2} u_{\text {out } 2} Q_{\text {out } 2}+\sum \tau,
\end{gathered}
$$

где $\Sigma=\pi d_{m} l-$ площадь смешивающей камеры с диаметром $d_{m}$ и длиной $\ell, \mathrm{m}^{2} ; S_{\text {out } 2}=\pi d_{m}^{2} / 4-$ площадь поперечного сечения смешивающей камеры, $\mathrm{m}^{2}$,

$$
\tau=\frac{1}{2} f \rho_{\text {out } 2} u_{\text {out } 2}{ }^{2}, f=0.316 \operatorname{Re}^{-0.25}, \operatorname{Re}=\frac{u_{\text {out } 2} d_{m} \rho_{\text {out } 2}}{\mu},
$$

где $\mu$ - коэффициент динамической вязкости многофазной смеси, Па.с:

$$
\mu=\frac{\left(w c \mu_{w}+(1-w c) \mu_{o}\right) Q_{\text {lout 2 }}+\mu_{g} Q_{\text {gout 2 }}}{Q_{\text {lout 2 }}+Q_{\text {gout 2 }}},
$$

$\tau$ - среднее значение касательного напряжения в смешивающей камере на стенке, Па.

Течение смеси в камере смешивания в условиях интенсивного перемешивания потоков жидкости и газа характеризуется значительной турбулентностью (8).

Задаем числовые параметры коэффициентов сопротивления соплового аппарата и диффузора при помощи экспериментальных зависимостей [11]. При расчетах принимают, что длина камеры смешения $\ell=7 d_{m}$ [11], а диаметр определяется как два диаметра сопла $d_{m}=2 d_{n}[1]$.

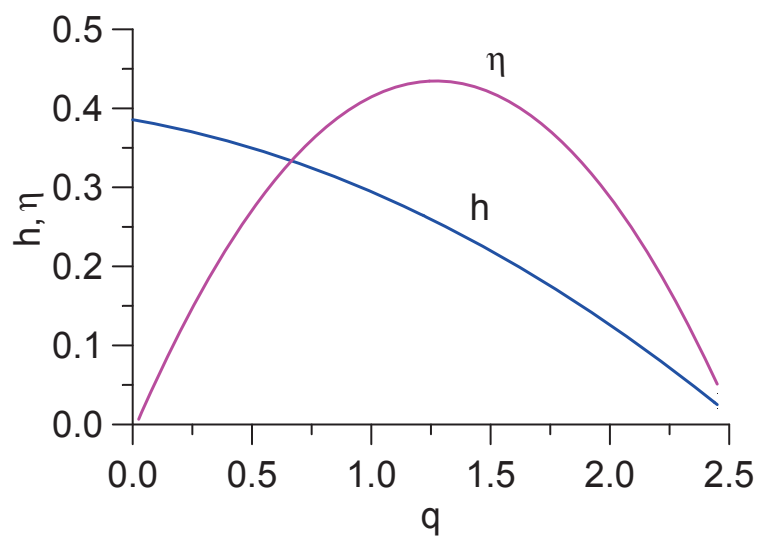

Puc. 4. Характеристики струйного аппарата

Fig. 4. Characteristics of the jet apparatus

Зависимости относительного напора струйного аппарата $h=\left(P_{\text {out } 1}-P_{\text {an in }}\right) /\left(P_{\text {in } 1}-P_{\text {an in }}\right)$ и его КПД $\eta=Q_{\text {in } 1} h /\left(Q_{\text {an in }}(1-h)\right)$ от относительного расхода
$q=Q_{\text {in } 1} / Q_{\text {an in }}$ получены расчетным путем при следующих входных параметрах: $P_{i n 1}=5 \mathrm{M \Pi а}, T_{i n 1}=T_{a n}$ ${ }_{\text {in }}=50{ }^{\circ} \mathrm{C}, Q_{\text {in } 1}=100 \mathrm{~m}^{3} /$ сут, газовый фактор $100 \mathrm{~m}^{3} / \mathrm{T}$, внутренний диаметр НКТ $62 \mathrm{~mm}$, диаметр сопла 5 мм, $\ell=70$ мм (длина смешивающей камеры), $P_{b s}=10 \mathrm{MПа} \mathrm{(давление} \mathrm{насыщения} \mathrm{свободным} \mathrm{га-}$ зом нефти), диаметр смешивающей камеры 10 мм (рис. 4). В интервале 0-2,5 изменение $q$ соответствует изменению давления в затрубном пространстве $P_{a n \text { in }}$ от 4,24 до 4,41 МПа. Из анализа графика видно, что максимальный коэффициент полезного действия (43\%) эжектора обеспечивается при значениях относительного расхода 1,3-1,5.

Примем условие, что технологические показатели нефтяной скважины известны, дебит по жидкости ограничен максимально допустимым содержанием затрубного газа на приеме насоса, а развиваемый напор не имеет ограничений. Предположим, что в насосе максимальное содержание свободного газа составляет после сепарации 20 \%, затрубное пространство добывающей скважины не связано с выкидной линией [12].

Смоделируем процесс работы установки со струйным аппаратом при исходных данных: $K=0,2 \mathrm{~m}^{3} /$ сут.МПа (коэффициент продуктивности), $d_{\text {well }}=130$ мм (диаметр обсадной колонны внутренний), $d_{\text {tube }}=73 \mathrm{Mм}, P_{\text {res }}=25,3 \mathrm{MПа} \mathrm{(пластовое} \mathrm{да-}$ вление), $P_{w h}=1,01 \mathrm{MПа} \mathrm{(устьевое} \mathrm{давление),} T_{w f}=80$ ${ }^{\circ} \mathrm{C}$ (температура пласта), $P_{b s}=12 \mathrm{MПа} \mathrm{(давление} \mathrm{на-}$ сыщения), $w c=50 \%$ (обводненность), $H_{\text {well }}=2500 \mathrm{M}$ (глубина вертикальной скважины), $H_{i n}=2000 \mathrm{~m}$ (глубина спуска ЭЦН).

Сопло струйного аппарата представляет собой канал, сужающийся в направлении течения пластовой жидкости. Наименьший перепад давлений пластовой жидкости на работу эжектора расходуется при определении оптимального диаметра соплового аппарата $[13,14]$. По формуле для нахождения выходного сечения сопла рассчитано оптимальное значение диаметра соплового аппарата $d_{n}=5 \mathrm{мm}$ при разнице давлений от 4,24 до 4,41 МПа $[13,14]$

Мощность, потребляемая УЭЦН, $N_{\text {tot }}$, динамический уровень $H_{d y n}$, давление в затрубном пространстве на устье скважины $P_{\text {an out }}$, коэффициент полезного действия струйного аппарата $\eta$, напор погружного насоса при пересчете на воду $H$ с учетом фиксированного значения диаметра сопла $d_{n}=5$ мм представлены в таблице. В зависимости от глубины спуска $H_{i n 1}$ потребляемую мощность определили по выражению:

$$
N_{\text {tot }}=\frac{\left(P_{\text {out }}-P_{\text {in }}\right) Q_{\text {liq }}}{864 D_{\text {gas }} D_{\text {vis }} \eta_{\text {pow }} \eta_{\text {cable }} \eta_{\text {pump }}},
$$

где $\eta_{\text {cable }}=0,96, \eta_{\text {pump }}=0,5$ и $\eta_{\text {pow }}=0,85-$ коэффициенты, которые учитывают мощностные потери соответственно в кабеле, насосе и двигателе.

Полученные результаты соответствуют максимальному дебиту $34 \mathrm{~m}^{3}$ /сут. С увеличением глубины спуска эжектора по расчетам увеличился динамический уровень и давление газа в затрубном 
пространстве, а номинальный напор и потребляемая мощность погружного насоса уменьшились [15].

таблица. Паралетры тандемной установки «электроцентробежный насос - струйный аппарат» для $d_{n}=5$ м.

Table. Tandem installation options «electric centrifugal pump - jet apparatus» for $d_{n}=5 \mathrm{~mm}$

\begin{tabular}{|c|c|c|c|c|}
\hline \multicolumn{5}{|c|}{ Параметры установки/Installation options } \\
\hline 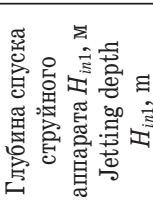 & 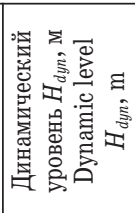 & 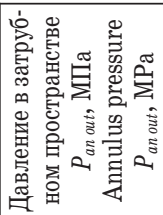 & 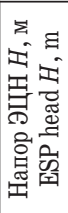 & 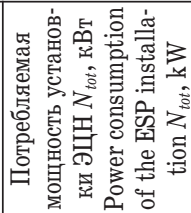 \\
\hline 80 & 1603 & 1,2 & 1680 & 16,1 \\
\hline 100 & 1622 & 1,3 & 1669 & 16,0 \\
\hline 200 & 1719 & 1,8 & 1624 & 15,5 \\
\hline 300 & 1817 & 2,4 & 1593 & 15,2 \\
\hline 400 & 1923 & 3,0 & 1567 & 15,0 \\
\hline 470 & 1998 & 3,5 & 1551 & 14,8 \\
\hline
\end{tabular}

Устойчивый режим работы погружной установки обеспечивается при максимальном дебите в определенном интервале глубины установки струйного аппарата (от 80 до 470 м в данном примере). Нижнее ограничение при незначительных глубинах спуска возникает из-за создания избыточного разрежения, ведущего к резкому падению КПД эжектора и расхода, а уменьшение глубины спуска $H_{i n 1}$ ведет к прекращению инжекции нефтяного газа в подъемных трубах. Верхний предел связан с повышением динамического уровня до глубины, на которую спускается погружная установка. При максимальных глубинах спуска эжектора могут наблюдаться периодически прорывы объемов скопившегося газа на приеме центробежного насоса из затрубного пространства скважины. При снижении подачи погружного насоса можно увеличить интервал глубины спуска, в рассмотренном примере при дебите жидкости $20,2 \mathrm{~m}^{3} /$ сут и газосодержании $1 \%$ он находится в диапазоне 50-1300 м $[16,17]$.

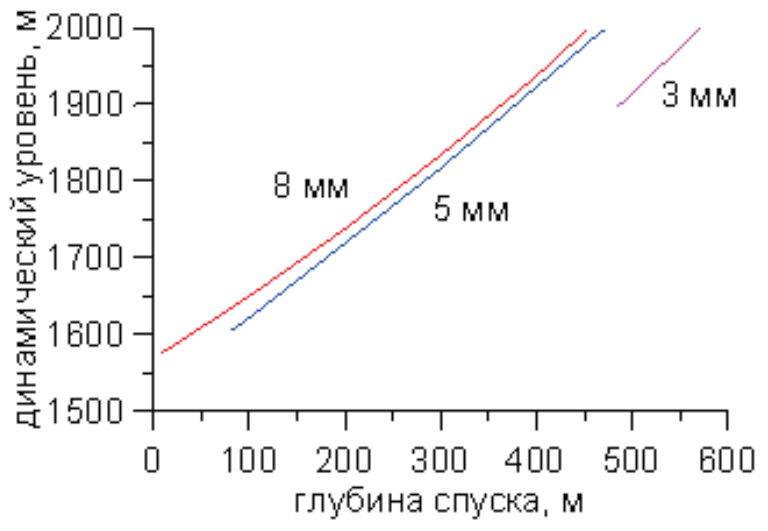

Pис. 5. Зависимость глубины спуска эжектора от динамического уровня для сопел с различными диаметрами

Fig. 5. Dependence of ejector descent depth on dynamic level for nozzles with different diameters
За счет подбора диаметра сопла можно при предельном дебите и фиксированной глубине спуска эжектора сделать работу УЭЦН более устойчивой [18-20]. Для конкретных диаметров сопел: 3,5 и 8 мм на рис. 5 показана зависимость динамического уровня в затрубном пространстве от глубины спуска эжектора. Анализ графиков показывает, что для устойчивой работы погружной установки диапазон изменения глубины установки струйного аппарата повышается и перемещается в направлении уменьшения глубины при увеличении диаметра соплового устройства.

Определенный расчетным путем дебит пластовой жидкости (34 м 3 сут) установки, которая оборудована струйным аппаратом с соплом, имеющим диаметр 5 мм, находится в интервале спуска эжектора 75-480 м. Поэтому выбранный диаметр сопла 5 мм для данного случая определяется в области наиболее оптимальных благоприятных параметров скважины.

На рис. 6 показаны интервалы работы в устойчивом режиме установки с эжектором, имеющим диаметр сопла 5 мм, обеспечивающие максимальный дебит жидкости. Анализ графических зависимостей показывает, что при выборе оптимальной глубины спуска эжектора учитывают различные изменения параметров нефтяной скважины во времени.

Процесс моделирования совместной работы установки и струйного аппарата является сложной задачей. Рассмотрим два предельных случая. В первом случае предполагается, что определенная часть газа удаляется в выкидную линию из затрубного пространства быстро. На рис. 7 показаны результаты расчета для сопла диаметром 5 мм номинального напора насоса для требуемой глубины и нижней линии интервала глубин спуска струйного аппарата в зависимости от количества сепарированного газа, перепускаемого в выкидную линию, при устойчивом режиме работы установки со струйным аппаратом. Анализ рисунков показывает, что номинальный напор центробежного насоса возрастает, а нижняя линия глубины спуска эжектора при увеличении количества скопившегося газа перемещается к направлению устья скважины, так как уменьшается доля газа в колонне НКТ и, соответственно, увеличиваются градиенты давлений.

Во втором предельном случае для решения задачи предполагается, что устьевое давление скважины является известным. Тогда при фиксированной глубине спуска струйного аппарата давление $P_{\text {an in }}$ (рис. 1) определяется из законов гидростатики, а задача заключается в определении оптимальных параметров установки с эжектором, при этом объем газа в приемной камере эжектора меньше объема газа отсепарированного.

В соответствии с расчетами режим устойчивой работы установки с эжектором при определенном значении $P_{\text {an out }}$ обеспечивается в узком интервале глубин спуска эжектора. Для сопла $d=5$ мм и рас- 


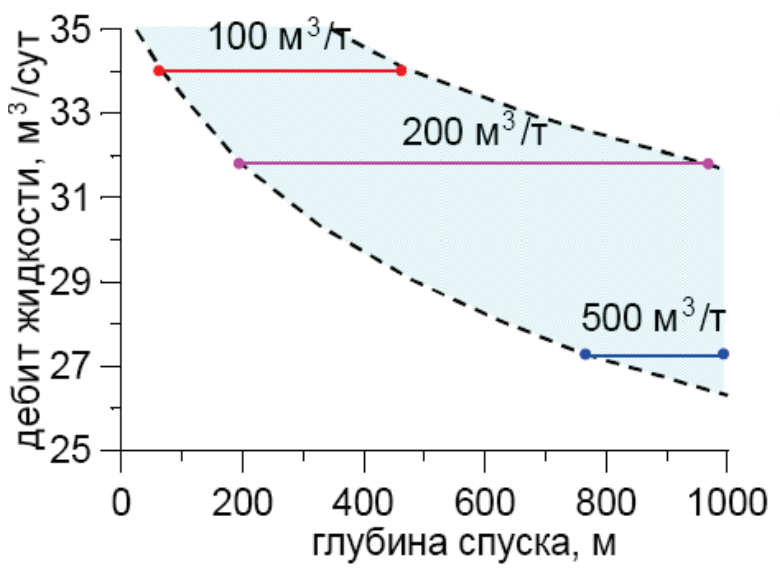

a)

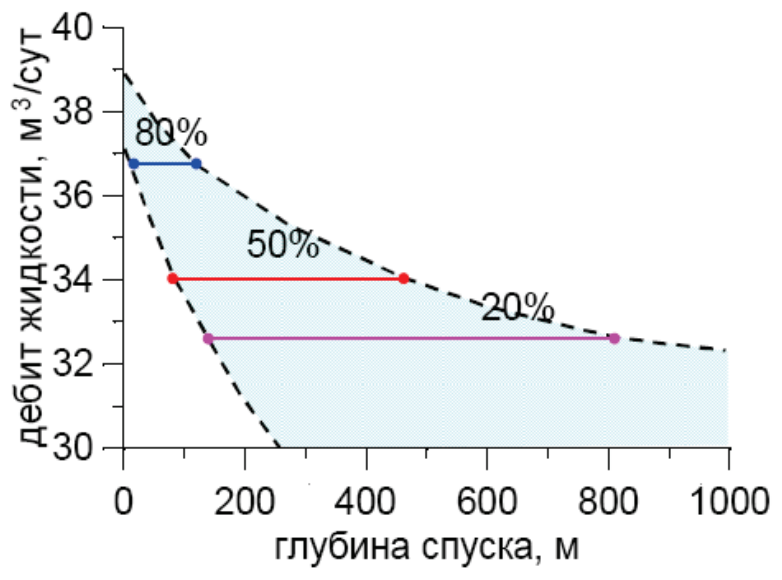

B)

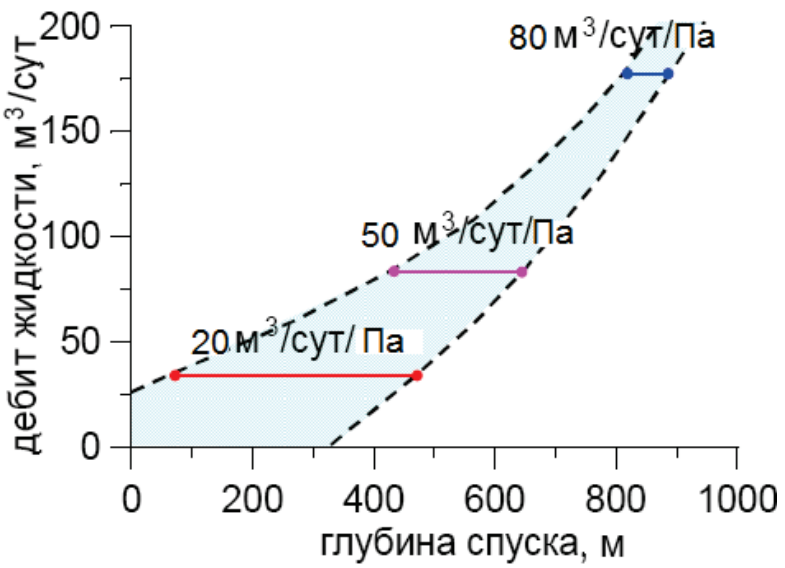

б)

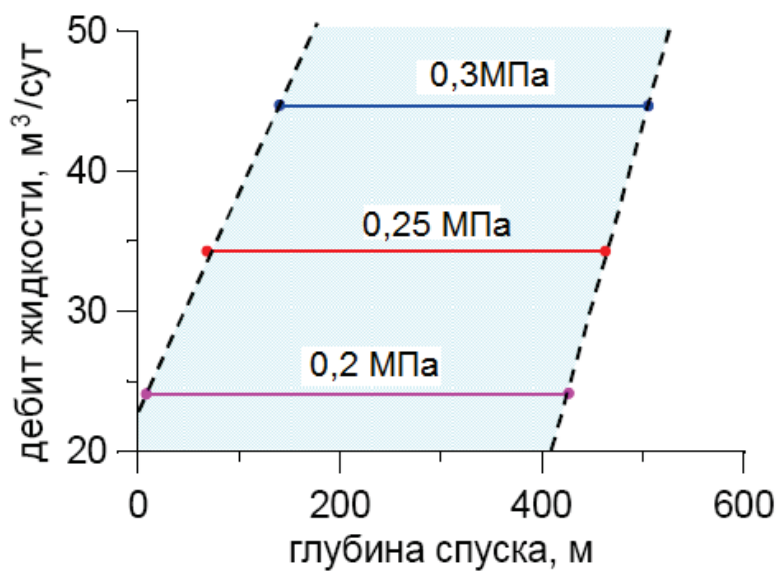

г)

Pис. 6. Интервалы эффективной работы погружной установки с эжекторол с диалетрол сопла 5 мл при значениях: а) газового фактора; б) коэффициента продуктивности; в) обводненности пластовой жидкости; г) давления пласта

Fig. 6. Intervals for efficient operation of a submersible installation with an ejector with a nozzle diameter of $5 \mathrm{~mm}$ with values: a) gas factor; б) productivity factor; 8 ) formation water cut; 2) formation pressure

считанных параметров данные интервалы составляют: для $P_{\text {an out }}=1,5 \mathrm{M \Pi а} 3 \mathrm{м}\left(H_{\text {in1 }}=139-142 \mathrm{~m}\right)$, для $P_{\text {an out }}=2,0 \mathrm{M \Pi а} 2 \mathrm{~m}\left(H_{\text {in1 }}=231-232 \mathrm{м}\right)$, для $P_{\text {an out }}=3,0$ МПа 1 м $\left(H_{\text {in1 }}=395 \mathrm{м}\right)$. Интервалы увеличиваются до нескольких десятков метров за счет изменения исходных величин (диаметр сопла, обводненность пластовой жидкости, газовый фактор). Повышенная чувствительность эжектора к изменению глубины спуска обусловлена тем, что в устойчивом режиме работы струйного аппарата должны соблюдаться неравенства одновременно: $Q_{g}<Q_{g \text { sep }}$, где $Q_{g \text { sep }}$ - объем отсепарированного газа на приеме погружного насоса, и $P_{a n}>P_{i n 2}$. На рис. 8 представлена схема эжектора с колонной НКТ.

Предполагаем, что затрубное пространство не связано с выкидной линией, тогда монотонный рост давления $P_{1}$ и увеличение динамического уровня $D$ будет происходить по мере накопления газа в затрубном пространстве скважины. Через впускной клапан будет поступать в эжектор из затрубного пространства газ. При превышении максимального значения давления $P_{1 c r}$ впускной клапан сработает. Тогда установится такой режим работы, при котором прекращается рост давления га- за $P_{1}$ и стабилизируется значение динамического уровня: объем газа из эжектора уравновесится таким же объемом газа из затрубного пространства. Основные параметры процесса будут зависеть от места установки эжектора в НКТ. Пусть забойное давление $P^{*}$ и расход жидкости $Q$ остаются постоянными параметрами. В таком случае из гидростатического закона распределения давления от забоя нефтяной скважины до места приема погружного насоса можно определить приближенное значение давления на приеме электроцентробежного насоса [13]:

$$
\frac{d P}{d x}=\rho_{L}(1-\Gamma(P)) g,\left.P\right|_{x=Y}=P^{*},\left.P\right|_{x=L}=P_{i n},
$$

где $\rho_{L}-$ плотность пластовой жидкости (без газа), кг $/ \mathrm{M}^{3} ; \Gamma$ - газовый фактор; $g$-ускорение свободного падения, $\mathrm{m}^{2} / \mathrm{c} ; P_{\text {in }}$ - давление, установившееся на приеме насоса, Па.

$$
\Gamma(P)=\Gamma_{\max }-\frac{\Gamma_{\text {max }} P}{P_{s}}=\frac{\Gamma_{\text {max }}\left(P_{s}-P\right)}{\bigoplus_{s}},
$$

где $P$ - определенное давление, Па; $P_{s}$ - давление насыщения газом нефти, Па. 


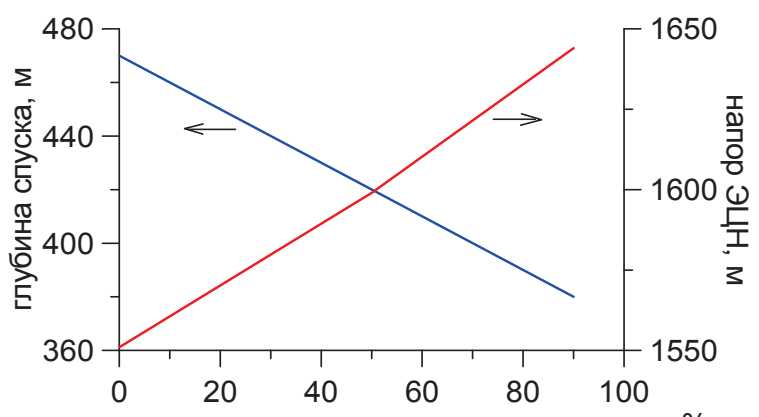

доля газа, поступающая в выкидную линию, \%

Puc. 7. Изменение номинального напора погружного насоса и нижней линии интервала глубин спуска эжектора в зависи мости от объема газа из выкидной линии

Fig. 7. Change of nominal pressure of submersible pump and lower line of the interval of the ejector descent depth depending on the volume of gas from the flow line

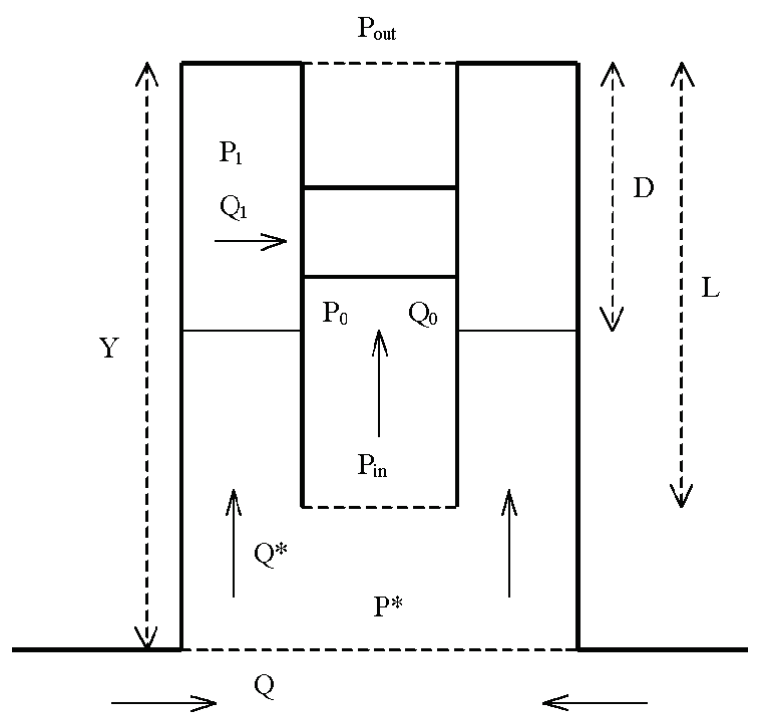

Puс. 8. Схема эжектора с колонной НКТ

Fig. 8. Scheme of the ejector with a tubing string

Проинтегрируем уравнение (9) с учетом линейной зависимости газового фактора Г от давления:

$$
P_{\text {in }}=\frac{1}{A_{1}}\left(-A_{2}+\left(A_{1} P^{*}+A_{2}\right) \exp \left(-A_{1} \rho_{L} g(Y-L)\right)\right) \text {, }
$$

где $A_{1}=\Gamma_{\max } / P_{S}, \Pi^{-1} ; A_{2}=1-\Gamma_{\max }-$ параметры уравнения состояния ГЖС; $L$ - глубина установки погружного насоса, м; $Y$ - глубина скважины, м.

В том случае, когда известны место установки эжектора $H$ и рабочий перепад давлений центробежного насоса $\Delta P$, можно с помощью зависимости из гидростатики определить давление на входе в эжектор [13]:

$$
P_{0}=\frac{1}{A_{1}}\left(\begin{array}{l}
-A_{2}+\left(A_{1}\left(P_{i n}+\Delta P\right)+A_{2}\right) \times \\
\times \exp \left(-A_{1} \rho_{L} g(L-H)\right)
\end{array}\right) .
$$

Расход пластовой жидкости определится равенством:

$$
Q_{0}=\frac{1-\Gamma\left(P^{*}\right)}{1-\Gamma\left(P_{0}\right)} Q
$$

где $Q$ - расход жидкости на забое скважины, $\mathrm{M}^{3} /$ сут; $P_{0}$ - давление пластовой жидкости на приеме эжектора, Па; $P^{*}$ - давление на забое скважины, Па.

При применении закона изменения гидростатического давления для условий затрубного пространства скважин при $L \leq x \leq D$ величину динамического уровня $D$ можно выразить из следующей формулы:

$$
\frac{d P}{d x}=\rho_{L}(1-\Gamma(P)) g,\left.P\right|_{x=D}=P_{1},\left.P\right|_{x=L}=P_{i n},
$$

тогда

$$
D=L-\frac{1}{\rho_{L} g A_{1}} \ln \left(\frac{A_{1} P_{\text {in }}+A_{2}}{A_{1} P_{1}+A_{2}}\right) .
$$

Устьевое давление рассчитывается из уравнения:

$$
P_{\text {out }}=\frac{1}{A_{1}}\left(-A_{2}+\left(A_{1} P_{4}+A_{2}\right) \exp \left(-A_{1} \rho_{\mathrm{L}} g H\right)\right) .
$$

При устойчивой работе скважины с установленным эжектором необходимо выполнение условий, определяемых ограничениями рабочих характеристик центробежного насоса и эжектора:

1) $\Gamma\left(P_{i n}\right)<\Gamma_{1}, \Gamma\left(P_{0}\right)<\Gamma_{2}-$ количество газа на приеме центробежного насоса и струйного аппарата не должно быть больше, чем предельно допустимые значения для каждого из них;

2) $P_{1}>P_{2}$ - для попадания свободного газа из затрубного пространства в эжектор его давление должно превышать давление струи пластовой жидкости;

3) $Q_{1} / Q_{0}<q_{c r}$ - существует ограничение расхода жидкости при максимальном значении газа в эжекторе;

4) $H<D$ - необходимо, чтобы динамический уровень был всегда больше глубины установки эжектора;

5) $P_{0}>P_{0 c r}-$ давление на входе в эжектор при определенном расходе $Q_{0}$ должно быть достаточным для предотвращения образования вакуума внутри эжектора.

Место установки эжектора рассчитывалось для двух величин объемного расхода: $Q=430 \mathrm{~m}^{3} /$ сут и $Q=260 \mathrm{~m}^{3}$ /сут. Параметры скважины выбраны следующим образом: $Y=3000 \mathrm{M}, \quad L=2000 \mathrm{м}$, $P^{*}=15 \mathrm{M \Pi а}, \quad Q^{*}=86 \mathrm{~m}^{3} /$ сут $, \quad Z=1,56 \cdot 10^{5} \mathrm{~m}^{2} / \mathrm{c}^{2}$, $\Gamma_{\max }=0,5, P_{S}=20 \mathrm{M \Pi а}, \rho_{L}=800 \kappa \Gamma / \mathrm{M}^{3}, \Delta P=2 \mathrm{M \Pi а}$, $S_{\text {out }}=0,005 \mathrm{~m}^{2}$; эжектор имеет геометрические характеристики: $d_{0}=d_{4}=60 \mathrm{MM}, \quad d_{S}=d_{20}=12 \mathrm{Mм}$, $d_{3}=30 \mathrm{MM}, \alpha_{i}=1, \beta_{i}=1, \xi_{i j}=\tau=0$.

На рис. 9 определяются зависимости по методике для нахождения установки эжектора, при этом можно для необходимого диапазона величин динамического уровня определить соответствующее ему значение глубины погружения эжектора.

Методика позволяет рассчитать глубину установки эжектора по известным параметрам скважины, центробежного насоса и эжектора по требуемым параметрам, таким как: обеспечение интервала изменения или необходимых значений да- 

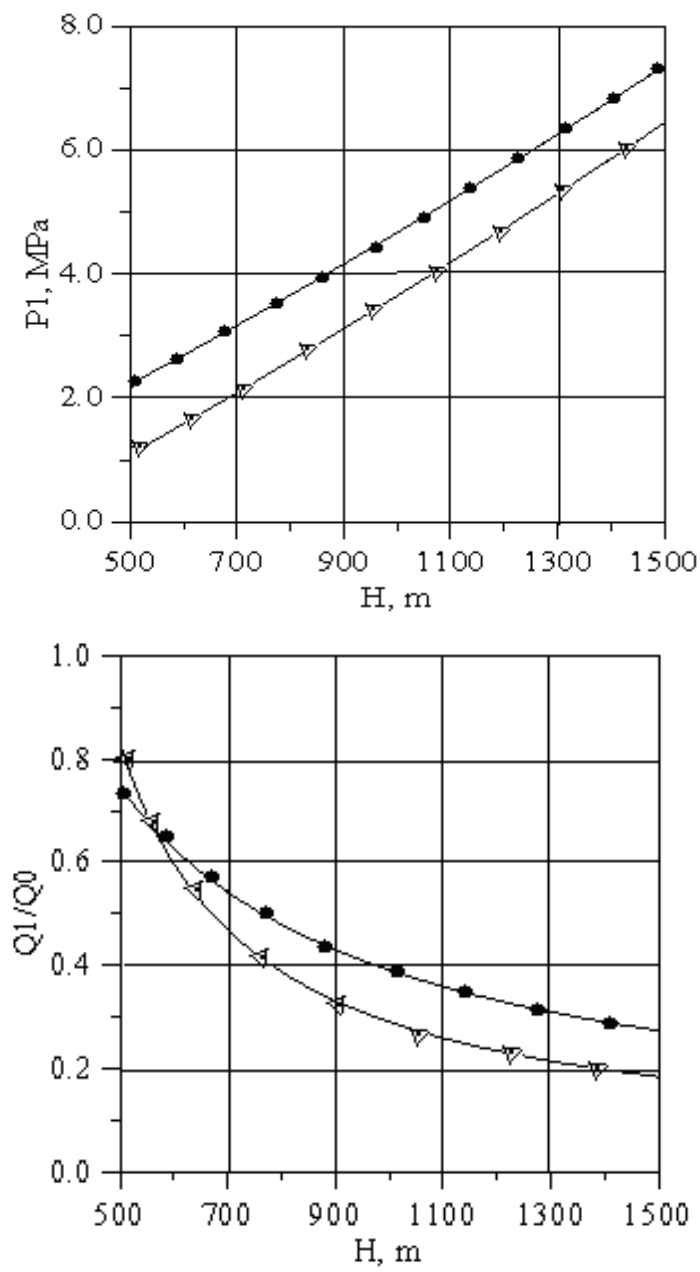
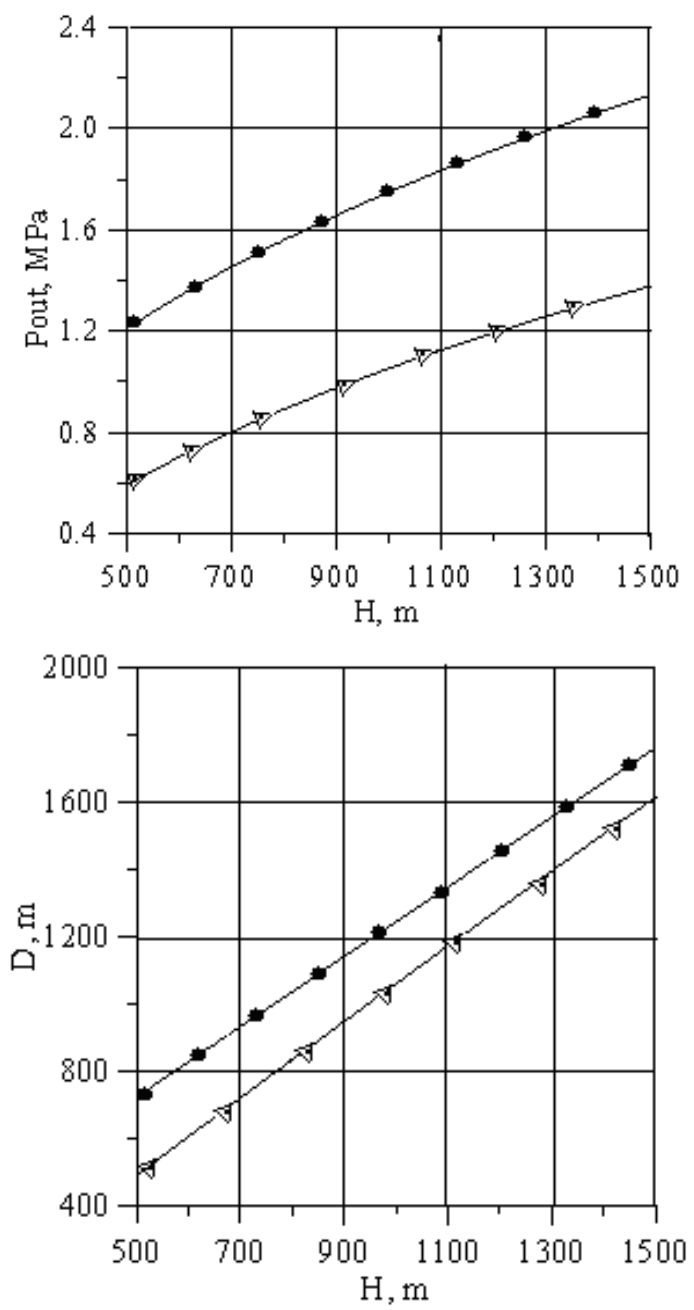

Pис. 9. Зависилости технологических параметров скважины от места установки эжектора при различных значениях относительного расхода Q. $\longrightarrow-Q=260 \mathrm{~m}^{3} / \mathrm{cym} ; \Delta \Delta \Delta-Q=430 \mathrm{~m}^{3} / \mathrm{cym}$

Fig. 9. Dependence of well technological parameters on ejector installation site at different values of the relative flow Q. $\bullet \bullet-$ $Q=260 \mathrm{~m}^{3} /$ day; $\Delta \Delta \Delta-Q=430 \mathrm{~m}^{3} /$ day

влений на устье, динамического уровня, давления на приеме эжектора и др.

\section{Выводы}

При проектировании оптимального режима работы скважины по технологии «электроцентробежный насос - струйный аппарат» предварительно определяются величины (например, диаметр

\section{СПИСОК ЛИТЕРАТУРЫ}

1. Лось Д.В. Анализ эксплуатации скважин тандемными глубинно-насосными установками в условиях высокой обводненности и газового фактора // Проблемы разработки месторождений углеводородных и рудных полезных ископаемых. - 2017. № 1. - C. 172-175.

2. Kuisheng W., Zhongqiang T. Revised characteristics curve optimizes jet pumping // Oil \& Gas Journal. - 2001. - V. 99. № $26 .-\mathrm{P} .52$.

3. Коротков В.В., Маклачков А.Н., Шитохина О.Г. Особенности работы струйных аппаратов // Научный альманах. - 2016. № 10-3 (24). - C. 178-182. сопла эжектора и глубина спуска) при необходимом изменении технологического режима нефтедобывающей скважины для обеспечения процесса инжекции скопившегося газа из затрубного пространства. Разработана методика для расчёта места установки эжектора с учетом известных характеристик скважины, погружного насоса и эжектора.

4. Brill J.P., Richardson M. Multiphase Flow in wells. - Texas: Society of Petroleum Engineers, 1999. - $384 \mathrm{p}$.

5. Квенцель А.Л., Ремень В.И. Пути повышения эффективности работы жидкостных струйных насосов // Вестник Луганского национального университета им. Владимира Даля. - 2017. № 3(5). - C. 45-52.

6. Duke S.E. The laid-back, adaptable hydraulic down hole pump // Drilling. -1982 . - V. 43. - № 3. - P. 84-95.

7. Методика расчета параметров струйного насоса при совместной эксплуатации с ЭЦН / А.С. Топольников, К.Р. Уразаков, Р.И. Вахитова, Д.А. Сарачева // ЭНЖ «Нефтегазовое дело». 2011. - № 3. - C. 134-146. 
8. Гилаев Г.Г., Бахтизин Р.Н., Уразаков К.Р. Современные методы насосной добычи нефти. - Уфа: Восточная печать, 2016. $410 \mathrm{c}$.

9. A Comprehensive Mechanistic Model for Two-Phase Flow in Wellbores / A.M. Ansari, N.D. Sylvester, C. Sarica, 0. Shoham, J.P. Brill // SPE Production \& Facilities. - 1994. - V. 9. - № 2. - P. 143-152.

10. Hasan A.R., Kabir C.S. Two-phase flow in vertical and inclined annuli // Int. J. Multiphase Flow. - 1992. - V. 18. - P. 279-293.

11. Сазонов Ю.А., Сазонова Р.В. Расчеты струйных насосов. - М.: ГАНГ, 1997. -52 c.

12. 0 снижении давления газа в затрубном пространстве скважин, оборудованных погружными центробежными насосами / Cарачева Д.А., Вахитова Р.И., Давлетов М.Ш. // Академический журнал Западной Сибири. - 2013. - Т. 9. - № 6 (49). - С. 38.

13. Расчет и подбор устьевого эжектора для скважин, оборудованных электроцентробежными установками / К.Р. Уразаков, Р.И. Вахитова, А.С. Топольников, К.А., Дубовицкий Р.А. Ахметшин // ЭНЖ «Нефтегазовое дело». - 2013. - № 4. C. 212-214.

14. Петри Н.Л., Вильсон П.М., Смарт Э.Э. Струйные насосы для нефтяных скважин // Нефть, газ и нефтехимия за рубежом / Пер. изд. журналов США. - 1983. - № 11.5-12. - С. 5-12.

15. Струйный аппарат для перепуска затрубного газа: пат. Рос. Федерация № 2517287; опубл. 27.05.2014, Бюл. № 15.
16. Калачев В.В. Экспериментальная оценка эффективности рабочего процесса струйных насосов, работающих в газо-жидкостном режиме, и перспективы их использования в природоохранном оборудовании // Гидравлика. - 2017. - № 2 (4). C. $93-130$.

17. Carvalho P.M., Podio A.L., Sepehrnoorik H.B. Modeling a Jet Pump with an Electrical Submersible jet Pump for Gassy Oil Well // J. of Petroleum Technology. - September 1998. - P. 34-36. DOI: 10.2118/48934-MS

18. The design, manufacture and testing of a jet-pump chiller for air conditioning and industrial application / I.W. Eames, A. Milazzo, D. Paganini, M. Livi // Applied Thermal Engineering. - 2013. V. 58. - № 1-2. - P. 234-240.

19. An electron jet pump: the Venturi effect of a Fermi liquid / D. Taubert, G.J. Schinner, C. Tomaras, S. Ludwig, H.P. Tranitz, W. Wegscheider // Journal of Applied Physics. - 2011. V. 109. - № 10. - P. 102-112.

20. Computational fluid dynamic analysis and design optimization of jet pumps / J. Fan, J. Eves, H.M. Thompson, V.V., Toropov N., Kapur D., Copley A. Mincher // Computers \& Fluids. - 2011 V. 46. - № 1. - P. 212-217. DOI: 10.1016/j.compfluid.2010.10.024

Поступила 12.02.2019 2.

\section{Информация об авторах}

Сарачева Д.А., кандидат технических наук, старший преподаватель кафедры электро- и теплоэнергетики Альметьевского государственного нефтяного института.

Baxuтова P.И., кандидат технических наук, доцент кафедры электро- и теплоэнергетики Альметьевского государственного нефтяного института.

Уразаков $\boldsymbol{K} . \boldsymbol{P . , ~ д о к т о р ~ т е х н и ч е с к и х ~ н а у к , ~ п р о ф е с с о р ~ к а ф е д р ы ~ м а ш и н ~ и ~ о б о р у д о в а н и я ~ н е ф т е г а з о в ы х ~ п р о м ы - ~}$ слов Уфимского государственного нефтяного технического университета. 


\title{
CALCULATION OF PARAMETERS OF JET APPARATUS FOR OPTIMAL JOINT WORK MODE WITH INSTALLATION OF ELECTRIC CENTRIFUGAL PUMP
}

\author{
Diana A. Saracheva', \\ sarachevadiana85@mail.ru \\ Roza I. Vakhitova', \\ roza-w@mail.ru \\ Kamil R. Urazakov², \\ UrazakK@mail.ru \\ 'Almetyevsk State Oil Institute,
2, Lenin street, Almetyevsk, 423450, Russia.
${ }^{2}$ Ufa State Oil Technical University,
1, Kosmonavtov street, Ufa, 450062, Russia.
}

The relevance of the research is caused by the need to solve the problem of removal of gas accumulating in the annular space of oil producing wells at the inlet of a submersible centrifugal pump. Excessive amount of free gas in the space between the casing and tubing contributes to formation of hydrate plugs, reducing the dynamic level in the well, as a result of which a supply failure may occur and complete stop of oil production is possible. For pumping free gas, it is proposed to use a jet apparatus for joint operation with an electric centrifugal pump, while it is important to correctly calculate the location of the ejector in the well and the diameter of the nozzle. The aim of the research is to develop and propose a method for calculating the parameters of the jet apparatus for the optimal mode of joint operation with an electric centrifugal pump.

Object: jet apparatus for removing annular gas in wells equipped with electrical centrifugal pump installations and high gas factor.

Methods. The problem posed was solved on the basis of statistical analysis of production data using information modern methods of processing results. The method of mathematical modeling was used when calculating the basic parameters of the jet apparatus to ensure the optimal mode when working together with the installations of electrical centrifugal pumps.

Results. The authors have proposed the mathematical model of an electric centrifugal pump together with a jet apparatus designed for pumping annular gas, as a result of which analytical dependencies are obtained that determine the optimal parameters and calculated values of the jet apparatus that ensure the stable operation of the "electrical centrifugal pump - jet apparatus» system. The authors developed the technique for calculating the location of the ejector in a well operated by an electric centrifugal pump.

\section{Key words:}

Jet apparatus, gas-liquid mixture, annular space, electric centrifugal pump, free gas, ejector, formation fluid, tubing.

\section{REFERENCES}

1. Los D.V. Analiz ekspluatatsii skvazhin tandemnymi glubinno-nasosnymi ustanovkami v usloviyakh vysokoy obvodnennosti i gazovogo faktora [Analysis of well operation by tandem deep well pumping units under conditions of high water content and gas factor]. Problems of development of hydrocarbon and ore mineral deposits, 2017, no. 1, pp. 172-175.

2. Kuisheng W., Zhongqiang T. Revised characteristics curve optimizes jet pumping. Oil \& Gas Journal, 2001, vol. 99, no. 26, p. 52.

3. Korotkov V.V., Maklachkov A.N., Shitokhina O.G. Osobennosti raboty struynykh apparatov [Features of the jet apparatus]. Scientific Almanac, 2016, no. 10-3 (24), pp. 178-182.

4. Brill J.P., Richardson M. Multiphase Flow in wells. Texas, Society of Petroleum Engineers, 1999. 384 p.

5. Quentzel A.L., Remen V.I. Puti povysheniya effektivnosti raboty zhidkostnykh struynykh nasosov [Ways to improve the efficiency of liquid jet pumps]. Bulletin of Lugansk National University. Vladimir Dahl., 2017, no. 3 (5), pp. 45-52.

6. Duke S.E. The laid-back, adaptable hydraulic down hole pump. Drilling, 1982, vol. 43, no. 3, pp. 84-95.

7. Topolnikov A.S., Urazakov K.R., Vakhitova R.I., Saracheva D.A. Metodika rascheta parametrov struynogo nasosa pri sovmestnoy ekspluatatsii s ETSN [The method of calculating the parameters of the jet pump when used together with the ESP]. Oil and Gas Business, 2011, no. 3, pp. 134-146.
8. Gilaev G.G., Bakhtizin R.N., Urazakov K.R. Sovremennye metody nasosnoy dobychi nefti [Modern methods of pumping oil production]. Ufa, Vostochnaya pechat Publ., 2016. 410 p.

9. Ansari A.M., Sylvester N.D., Sarica C., Shoham O., Brill J.P. A Comprehensive Mechanistic Model for Two-Phase Flow in Wellbores. SPE Production \& Facilities, 1994, vol. 9, no. 2, pp. 143-152.

10. Hasan A.R., Kabir C.S. Two-phase flow in vertical and inclined annuli. Int. J. Multiphase Flow, 1992, vol. 18, pp. 279-293.

11. Sazonov Yu.A., Sazonova R.V. Raschety struynykh nasosou [Calculation of jet pumps]. Moscow, GANG Publ., 1997. 52 p.

12. Saracheva D.A., Vakhitova R.I., Davletov M.Sh. On the reduction of gas pressure in the annulus of wells equipped with submersible centrifugal pumps. Academic Journal of Western Siberi, 2013, vol. 9, no 6 (49), p. 38.

13. Urazakov K.R., Vakhitova R.I., Topolnikov A.S., Dubovitsky K.A., Akhmetshin R.A. Raschet i podbor ustyevogo ezhektora dlya skvazhin, oborudovannykh elektrotsentrobezhnymi ustanovkami [Calculation and selection of the wellhead ejector for wells equipped with electrical centrifugal installations]. Oil and Gas Business, 2013, no. 4, pp. 212-214.

14. Petri N.L., Wilson P.M., Smart E.E. Struynye nasosy dlya neftyanykh skvazhin [Jet pumps for oil wells]. Oil, gas and petrochemistry abroad. Translated by US magazines, 1983, no. 11-12, pp. $5-12$. 
15. Urazakov K.R., Vakhitova R.I., Saracheva D.A., Abramova E.V. Struyny apparat dlya perepuska zatrubnogo gaza [Jet apparatus for bypassing annular gas]. Patent RF, no. 2517287, 2014.

16. Kalachev V.V. Eksperimentalnaya otsenka effektivnosti rabochego protsessa struynykh nasosov, rabotayushchikh v gazo-zhidkostnom rezhime, i perspektivy ikh ispolzovaniya v prirodookhrannom oborudovanii [Experimental evaluation of the efficiency of the working process of jet pumps operating in gas-liquid mode, and the prospects for their use in environmental protection equipment]. Hydraulics, 2017, no. 2 (4), pp. 93-130.

17. Carvalho P.M., Podio A.L., Sepehrnoorik H.B. Modeling a Jet Pump with an Electrical Submersible jet Pump for Gassy Oil Well. J. of Petroleum Technology, September 1998, pp. 34-36. DOI: $10.2118 / 48934-\mathrm{MS}$
18. Eames I.W., Milazzo A., Paganini D., Livi M. The design, manufacture and testing of a jet-pump chiller for air conditioning and industrial application. Applied Thermal Engineering, 2013, vol. 58, no. 1-2, pp. 234-240.

19. Taubert D., Schinner G.J., Tomaras C., Ludwig S., Tranitz H.P., Wegscheider W. An electron jet pump: the Venturi effect of a Fermi liquid. Journal of Applied Physics, 2011, vol. 109, no. 10, pp. 102-112.

20. Fan J., Eves J., Thompson H.M., Toropov V.V., Kapur N., Copley D., Mincher A. Computational fluid dynamic analysis and design optimization of jet pumps. Computers \& Fluids, 2011, vol. 46, no. 1, pp. 212-217. D0I: 10.1016/j.compfluid.2010.10.024

Received: 12 February 2019.

\section{Information about the authors}

Diana A. Saracheva, Cand. Sc., senior lector, Almetyevsk State Oil Institute.

Roza I. Vakhitova, Cand. Sc., assistant professor, Almetyevsk State Oil Institute.

Kamil R. Urazakov, Dr. Sc., professor, Ufa State Oil Technical University. 